\title{
Retrohepatic Vena Cava Resection Associated With Right Trisectionectomy and Caudate Lobectomy for Colorectal Liver Metastases
}

\author{
Gerardo Sarno, MD, Hassan Z. Malik, MD, Stephen W. Fenwick, MD, Ali A. Al-Sarira, MBBS, \\ and Graeme J. Poston, MS
}

North Western Hepatobiliary Surgery Unit, University Hospital Aintree, Liverpool, UK

\begin{abstract}
Background. In case of colorectal liver metastases invading the retrohepatic vena cava (RHVC) and the hepatocaval confluence major liver resection and venous resection are the treatment of choice. The use of venovenous bypass is still a matter of debate. We describe our technique without the use of venovenous bypass for resection of a tumor invading the RHVC.

Methods. A 61-year-old woman was referred to us with a large colorectal liver metastases involving the right hemiliver and caudate lobe also involving the RHVC. Through a reverse-L incision, the liver was exposed. As a result of the RHVC involvement, the hanging maneuver was not attempted. The right liver was partially mobilized. Vascular control was achieved by encircling the supra and infrahepatic vena cava and hepatic pedicle. The right hepatic artery and right portal vein were divided before parenchymal transection with Cavitron Ultrasonic Surgical Aspirator (CUSA) (under intermittent Pringle maneuver). The middle hepatic vein was divided within the
\end{abstract}

Electronic supplementary material The online version of this article (doi:10.1245/s10434-010-1126-9) contains supplementary material, which is available to authorized users.

(C) Society of Surgical Oncology 2010

First Received: 12 February 2010;

Published Online: 12 June 2010

G. Sarno, MD

e-mail: gsarno79@yahoo.it parenchyma. This maneuver enabled us to swing the remnant liver and expose the RHVC. The caudate lobe was mobilized from left to right, leaving the right hepatic vein attached. This exposed the origin of the RHVC involvement. This was controlled by Satinsky clamp. A cuff of vena cava was resected and over sewn with 3-0 Prolene. Finally, the right hepatic vein was divided.

Results. Complete vascular control before resection and meticulous technique allowed a safe resection with minimal intraoperative blood loss. The patient was discharged on postoperative day 7 after an uneventful in-hospital stay. Conclusions. Complete vascular control of the liver before resection allows safe and controlled resection of tumour involving the RHVC without the need for venovenous bypass. However, such a technique should be limited to high-volume hepatobiliary centers. 